\title{
Association Between Evening Admissions and Higher Mortality Rates in the Pediatric Intensive Care Unit
}

\author{
Yeseli Arias, MD; Douglas S. Taylor, MD, PhD; and James P. Marcin, MD, MPH
}

\begin{abstract}
Background. Previous research investigating the relationship between the time of admission and mortality rates has yielded inconsistent results and has not been conducted in the pediatric intensive care unit (PICU) patient population.

Objective. To determine whether an association between the time of admission (weekday versus weekend and daytime versus evening) and the risk of death exists among pediatric patients included in a cohort of children admitted to a national sample of PICUs.

Design/Methods. We analyzed retrospectively a cohort of consecutive admissions to 15 PICUs included in the Pediatric Intensive Care Unit Evaluations database. The odds of death were analyzed by using mixed-effects, multivariate, logistic regression, with clustering at the hospital level. The primary independent variables were admission to the PICU on a weekend and admission to the PICU during evening hours. The severity of illness was adjusted by using the Pediatric Risk of Mortality III probability of death score.
\end{abstract}

Patients. All 20547 emergency PICU admissions made between May 1995 and December 2001 were included in the analyses.

Main Outcome Measures. The primary outcome was death within 48 hours after admission to the PICU.

Results. Pediatric patients admitted to the PICU during evening hours had higher odds of death (odds ratio [OR]: $1.28 ; 95 \%$ confidence interval [CI]: 1.00-1.62) than did those admitted during daytime hours. Subgroup analyses revealed higher odds of death among patients admitted with shock (OR: 4.09; 95\% CI: 1.65-10.1), with congenital cardiovascular disease (OR: 3.90; 95\% CI: 1.3711.1), or after cardiac arrest (OR: $1.80 ; 95 \%$ CI: 1.04-3.13). There was no association between mortality rates and the day of admission (weekend admissions versus weekday admissions).

Conclusions. An increased risk of death exists for some pediatric patients admitted to the PICU during evening hours. It remains necessary to determine whether this finding results from differences in the structure of care, processes of care, or both. Pediatrics 2004;113:e530-e534. URL: http://www.pediatrics.org/cgi/ content/full/113/6/e530; health care quality, PRISM, pediatrics, intensive care units, patient admission, mortality.

ABBREVIATIONS. ICU, intensive care unit; PICU, pediatric intensive care unit; PICUE, Pediatric Intensive Care Unit Evaluation; PRISM, Pediatric Risk of Mortality; OR, odds ratio; CI, confidence interval.

From the Department of Pediatrics, University of California, Davis, California.

Received for publication Sep 2, 2003; accepted Jan 28, 2004.

Reprint requests to (J.P.M.) University of California, Davis, Medical Center, 2516 Stockton Blvd, Sacramento, CA 95817. E-mail: jpmarcin@ucdavis.edu PEDIATRICS (ISSN 0031 4005). Copyright @ 2004 by the American Academy of Pediatrics.
I o improve the quality of care, discrepancies in health care delivery and their effects on patient outcomes must be identified. Recent research has demonstrated that, among large patient cohorts, differences in time of admission are associated with differences in patient outcomes. Bell and Redelmeier ${ }^{1}$ identified a significantly higher mortality rate among adult patients admitted on the weekend, compared with similar patients admitted on weekdays. Barnett et $\mathrm{al}^{2}$ in a study of $>150000$ adult patients admitted to 38 intensive care units (ICUs), demonstrated both a higher risk-adjusted odds of in-hospital death and a longer ICU length of stay for patients admitted on the weekend, compared with patients admitted on weekdays. Similarly, neonatal mortality rates were shown to be slightly higher among infants born on weekends and during evening hours, compared with those born on weekdays and during daytime hours, respectively. ${ }^{3-8}$ Whether an association exists between patient outcomes and time of admission in the pediatric patient population is unknown.

To address this question, the Pediatric Intensive Care Unit Evaluations (PICUEs) database, representing a cohort of 34993 pediatric ICU (PICU) patients from 15 institutions in the United States, was analyzed. ${ }^{9}$ The objectives of this study were to compare the risk-adjusted mortality rates for weekend admissions versus weekday admissions and the risk-adjusted mortality rates for evening admissions versus daytime admissions.

\section{METHODS}

\section{Patients}

Data for analysis were obtained from the most current available PICUEs database, Research Dataset 20-02.9 This database contains patient-level data from 34993 admissions to 15 PICUs in the United States. Details of the site-selection procedures for the 15 study sites, general data-collection methods, and other analyses of this data set have been published. ${ }^{9,10}$ Briefly, a minimum of 67 demographic, diagnostic physiologic, laboratory, and outcome data points were collected for all patients within 24 hours after admission to the PICU. Data for these analyses were collected for consecutive admissions between May 1995 and December 2001. Patients excluded from the database were patients admitted to the PICU for recovery from procedures usually cared for at other hospital locations, patients staying in the ICU $<2$ hours, and patients admitted in a state of continuous cardiopulmonary resuscitation. All data included in the database met quality standards, as assessed by data reabstraction and reliability testing.

The database includes data on 20547 emergency admissions and 14446 elective admissions, as defined by the Pediatric Risk of Mortality (PRISM)/PICUEs criteria. We limited our analyses, a priori, to patients admitted on an emergency basis, because we thought that their outcomes would be most sensitive to time 
variations in structure (eg, staffing levels) and processes (ie, the specific clinical activities performed) of care. ${ }^{11}$

\section{Study Design}

The primary outcome variable for these analyses was death within 48 hours after admission to the PICU. Only deaths that occurred within 48 hours after admission were considered, because such deaths were more likely to be attributable to differences during the initial hours after admission (eg, death resulting from shock is more sensitive to care during the initial 24 hours after admission, whereas death occurring days to weeks after admission is more likely to be attributable to complications of care or hospital-acquired infections). ${ }^{1,2,12}$ The primary independent variables were admission to the PICU on a weekend and admission to the PICU during the evening hours. Weekend admissions were defined as admissions to the PICU between 7 PM Friday and 7 АM Monday. Evening admissions were defined as admissions between 5 PM and 7 AM.

Other independent variables included as covariates were previous PICU admission during the current hospitalization, postoperative status, transfer from the inpatient ward, and use of vasoactive medications or ventilation within 24 hours after admission. The severity of illness was adjusted by using the PRISM III probability of death. ${ }^{9,10}$ PRISM III is a third-generation mortalityprediction algorithm based on individual patient demographic, physiologic, and laboratory data ${ }^{9,13}$ and is used to adjust for differences in mortality risk when comparing different populations of patients. The natural logarithm of the PRISM III probability of death was entered into the analyses as a quadratic transformation because of its curvilinear relationship with the observed logarithm of the odds of death.

Subgroup analyses were conducted on primary admission diagnoses according to the PRISM/PICUEs admission categories, including asthma, cancer, cardiac arrest, diabetic ketoacidosis, drug overdose, congenital cardiovascular disease, acquired cardiovascular disease, acute hypoxic ischemic encephalopathy, medical device malfunction, meningitis, pneumonia/bronchiolitis, seizure, sepsis, shock, suicide attempt, and trauma. These analyses were conducted to assess differences in mortality rates among individual diagnoses and were performed only for diagnoses with at least 100 patients and $\geq 10$ deaths. ${ }^{14,15}$ Primary admission diagnoses from PRISM/PICUEs that were not included were gastroesophageal reflux, human immunodeficiency virus, chromosomal abnormality, solid-organ transplantation, and "other."

\section{Analyses}

Univariate analyses of continuous variables were conducted with Student's $t$ test or the Mann-Whitney $U$ test, when appropriate. Univariate analyses of dichotomous variables were conducted with the $\chi^{2}$ test or Fisher's exact test, when appropriate. The odds of death within 48 hours were analyzed by using mixed-effects, multivariate, logistic regression, with clustering at the hospital level. ${ }^{16}$ Although hospital names were anonymous, each patient in the database was identified with a unique hospital code to allow statistical clustering of patients from the same institution. The adjusted odds ratios (ORs) are reported with 95\% confidence intervals (CIs). Statistical significance was defined as $P<.05$. All calculations were performed by using Stata version 7 software (Stata, College Station, TX).

\section{Human Subject Protection}

This study was reviewed and approved by the University of California, Davis, Office for Human Subjects Protection.

\section{RESULTS}

\section{Univariate Analyses}

There were 20547 pediatric patients admitted to the 15 PICUs on an emergency basis, and 7052 (34.3\%) were admitted on weekends (Table 1). Compared with patients admitted on weekdays, patients admitted on weekends were slightly older (6.1 vs 5.8 years, $P=.01$ ), less likely to be admitted postoperatively $(8.4 \%$ vs $10.2 \%, P=.01)$, less likely to have experienced a previous PICU admission (3.4\% vs $4.0 \%, P=.04)$, and less likely to have received vasoactive medications within 24 hours after admission (16.0\% vs $17.1 \%, P=.04)$. There was no difference between weekend and weekday admissions regarding the severity of illness (mean PRISM III scores of 5.7 vs $5.9, P=.12$ ).

Patients admitted during the evening $(n=12746$, $62 \%$ ), compared with those admitted during the daytime $(n=7801,38 \%)$, were more likely to be male (57.8\% vs 56.4\%, $P=.04$ ) and were older (6.1 vs 5.6 years, $P<.01$ ) but were less likely to have experienced a previous PICU admission (3.0\% vs 5.2\%, $P<$ $.01)$, received vasoactive medications within 24 hours after admission $(15.1 \%$ vs $19.5 \%, P<.01)$, or received mechanical ventilation within 24 hours after admission (34.5\% vs 38.4\%, $P<.01)$. Patients admitted during the evening demonstrated a lower mean PRISM III score, compared with those admitted in the daytime (mean PRISM III scores of 5.5 vs $6.3, P<$ .01) (Table 1).

Table 1 demonstrates that the unadjusted overall PICU mortality rates were not statistically different for patients admitted on weekends and patients admitted on weekdays $(4.71 \%$ vs $4.71 \%, P=.99)$. Similarly, there was no statistical difference in the rates of death within 48 hours after admission between the groups $(2.28 \%$ vs $2.17 \%, P=.61)$. As shown in Table 2 , the primary diagnoses for the weekend and weekday groups were trauma $(20 \%$ vs $16 \%, P<.01)$ and asthma $(10.0 \%$ vs $8.8 \%, P=.05)$. Overall, there were

TABLE 1. Baseline Patient Characteristics*

\begin{tabular}{|c|c|c|c|c|c|c|}
\hline & $\begin{array}{l}\text { Weekend } \\
\text { Admission } \\
(n=7052)\end{array}$ & $\begin{array}{c}\text { Weekday } \\
\text { Admission } \\
(n=13495)\end{array}$ & $P$ & $\begin{array}{c}\text { Evening } \\
\text { Admission } \\
(n=12746)\end{array}$ & $\begin{array}{c}\text { Daytime } \\
\text { Admission } \\
(n=7801)\end{array}$ & $P$ \\
\hline Overall mortality rate, $(\%)$ & $4.71(4.23-5.23)$ & $4.71(4.36-5.08)$ & .99 & $4.59(4.23-4.97)$ & $4.91(4.44-5.41)$ & .29 \\
\hline Death within $48 \mathrm{~h}(\%)$ & $2.28(1.95-2.66)$ & $2.17(1.93-2.43)$ & .61 & $2.24(1.99-2.52)$ & $2.15(1.84-2.50)$ & .67 \\
\hline Male, $(\%)$ & $57.4(56.3-58.6)$ & $57.2(56.4-58.0)$ & .74 & $57.8(57.0-58.7)$ & $56.4(55.3-57.5)$ & .04 \\
\hline Age, (y) & $6.1(5.9-6.2)$ & $5.8(5.7-5.9)$ & .01 & $6.1(6.0-6.2)$ & $5.6(5.5-5.8)$ & $<.01$ \\
\hline PRISM III-24 score & $5.7(5.5-5.9)$ & $5.9(5.7-6.0)$ & .12 & $5.5(5.4-5.6)$ & $6.3(6.1-6.4)$ & $<.01$ \\
\hline Previous PICU admission (\%) & $3.4(3.0-3.9)$ & $4.0(3.7-4.4)$ & .04 & $3.0(2.7-3.3)$ & $5.2(4.7-5.7)$ & $<.01$ \\
\hline Postoperative, (\%) & $8.4(7.7-9.1)$ & $10.2(9.65-10.7)$ & $<.01$ & $9.7(9.2-1.0)$ & $9.27(8.6-9.9)$ & .28 \\
\hline Transfer from ward, (\%) & $34.9(33.8-36.1)$ & $36.8(35.9-37.6)$ & .01 & $35.2(3.4-3.6)$ & $37.8(3.7-3.9)$ & $<.01$ \\
\hline Vasoactives within $24 \mathrm{~h},(\%)$ & $16.0(15.1-16.9)$ & $17.1(16.5-17.8)$ & .04 & $15.1(14.5-15.7)$ & $19.5(18.6-20.4)$ & $<.01$ \\
\hline Ventilation within $24 \mathrm{~h},(\%)$ & $35.2(34.1-36.4)$ & $36.4(35.5-37.2)$ & .11 & $34.5(33.7-35.3)$ & $38.4(37.3-39.5)$ & $<.01$ \\
\hline
\end{tabular}

* Weekend admission was defined as admission between 7 PM Friday and 7 AM Monday. Evening admission was defined as admission between 5 PM and 7 AM Monday through Sunday. Values are mean and 95\% CI. 


\begin{tabular}{|c|c|c|c|c|c|c|}
\hline & \multicolumn{6}{|c|}{ No. of Cases (\%) } \\
\hline & $\begin{array}{l}\text { Weekend } \\
\text { Admission } \\
(n=7052)\end{array}$ & $\begin{array}{l}\text { Weekday } \\
\text { Admission } \\
(n=13495)\end{array}$ & $P$ & $\begin{array}{c}\text { Evening } \\
\text { Admission } \\
(n=12746)\end{array}$ & $\begin{array}{l}\text { Daytime } \\
\text { Admission } \\
(n=7801)\end{array}$ & $P$ \\
\hline Asthma & $707(10.0)$ & $1192(8.8)$ & .05 & $1138(8.9)$ & $761(9.8)$ & .05 \\
\hline Cancer & $97(1.4)$ & $250(1.9)$ & .01 & $209(1.6)$ & $138(1.8)$ & .49 \\
\hline Cardiac arrest & $88(1.2)$ & $183(1.4)$ & .52 & $153(1.2)$ & $118(1.5)$ & $<.01$ \\
\hline Diabetic ketoacidosis & $253(3.6)$ & $519(3.8)$ & .36 & $440(3.5)$ & $332(4.3)$ & .52 \\
\hline Drug overdose & $253(3.6)$ & $377(2.8)$ & $<.01$ & $456(3.6)$ & $174(2.2)$ & $<.01$ \\
\hline Congenital cardiovascular disease & $222(3.1)$ & $719(5.3)$ & $<.01$ & $430(3.4)$ & & \\
\hline Acquired cardiovascular disease & $111(1.6)$ & $350(2.6)$ & $<.01$ & $254(2.0)$ & $511(6.6)$ & $<.01$ \\
\hline Acute HIE & $18(0.26)$ & $49(0.36)$ & .20 & $41(0.32)$ & $207(2.7)$ & $<.01$ \\
\hline \multirow[t]{3}{*}{ Medical device malfunction } & $107(1.5)$ & $217(1.6)$ & .62 & $205(1.6)$ & $26(0.33)$ & .89 \\
\hline & & & & & $119(1.5)$ & .64 \\
\hline & $177(2.5)$ & $284(2.1)$ & .06 & $283(2.2)$ & & \\
\hline Meningitis & $967(14)$ & $1867(14)$ & .81 & $1658(13)$ & $178(2.3)$ & .77 \\
\hline Pneumonia/bronchiolitis & $691(9.7)$ & $1137(8.4)$ & $<.01$ & 1187 (9.3) & $1176(15)$ & $<.01$ \\
\hline Seizure & $145(2.1)$ & $353(2.6)$ & .01 & $292(2.3)$ & $641(8.2)$ & $<.01$ \\
\hline Sepsis & $141(2.0)$ & $272(2.0)$ & .94 & $242(1.9)$ & $206(2.6)$ & .11 \\
\hline Shock & $57(0.8)$ & $111(0.8)$ & .91 & $124(1.0)$ & $171(2.2)$ & .15 \\
\hline Suicide attempt & $1435(20)$ & $2112(16)$ & $<.01$ & $2585(20)$ & $44(0.6)$ & $<.01$ \\
\hline Trauma & & & & & $962(12)$ & $<.01$ \\
\hline
\end{tabular}

* Weekend admission was defined as admission between 7 PM Friday and 7 AM Monday. Evening admission was defined as admission between 5 PM and 7 AM Monday through Sunday. HIE, hypoxic-ischemic encephalopathy.

proportionately more admissions because of trauma, asthma, seizures, and drug overdoses on weekends. On weekdays, proportionately more patients were admitted with the diagnoses of cancer, congenital cardiovascular disease, acquired cardiovascular disease, and sepsis.

In a comparison of evening admissions and daytime admissions, the unadjusted overall PICU mortality rates $(4.59 \%$ vs $4.91 \%, P=.29)$ and rates of death within 48 hours $(2.24 \%$ vs $2.15 \%, P=.67)$ were not statistically different. As shown in Table 2, the most common diagnoses for evening and daytime admissions were trauma $(20 \%$ vs $12 \%, P<.01)$ and pneumonia/bronchiolitis (13\% vs $15 \%, P<.01)$. In addition, there were proportionately more admissions because of asthma and congenital cardiovascular disease during the daytime, compared with the evening, but proportionately more admissions because of seizures and drug overdoses during the evening, compared with the daytime.

\section{Multivariate Analyses}

Mixed-effects, multivariate, hierarchical, logistic regression was used to adjust for age, gender, a quadratic transformation of the logarithm of the PRISM III probability of death, previous PICU admission, postoperative care status, patient transfer status, and the requirement for vasoactive medications or ventilation within 24 hours after admission. The odds of death within 48 hours after admission for patients admitted on weekends was not higher than that for patients admitted on weekdays, as shown in Table 3 (OR: 1.00; 95\% CI: 0.78-1.28). Furthermore, in the subgroup analyses there was no diagnosis with a different odds of death when weekend admissions were compared with weekday admissions (Table 4).

However, similar analyses demonstrated that pediatric patients admitted during evening hours had a higher odds of death than did patients admitted

TABLE 3. Results of Multivariate Hierarchical, Logistic Regression Predicting Death Within 48 Hours After Admission

\begin{tabular}{|c|c|c|c|c|c|c|}
\hline & \multicolumn{3}{|c|}{ Weekend Admissions } & \multicolumn{3}{|c|}{ Evening Admissions } \\
\hline & OR & $95 \% \mathrm{CI}$ & $P$ & OR & $95 \% \mathrm{CI}$ & $P$ \\
\hline Weekend admission & 1.00 & $0.78-1.28$ & .99 & & & \\
\hline Evening admission & & & & 1.28 & $1.00-1.62$ & .04 \\
\hline Age, (y) & 1.00 & $0.98-1.02$ & .74 & 1.00 & $0.98-1.02$ & .74 \\
\hline Male & 1.01 & $0.80-1.27$ & .96 & 1.00 & $0.79-1.26$ & .99 \\
\hline PRISM III prediction* & 2.38 & $2.23-2.54$ & $<.01$ & 2.38 & $2.23-2.54$ & $<.01$ \\
\hline${\text { (PRISM III prediction) }{ }^{2}+}$ & 0.96 & $0.94-0.98$ & $<.01$ & 0.96 & $0.94-0.98$ & $<.01$ \\
\hline Previous ICU admission $\ddagger$ & 0.52 & $0.30-0.89$ & .02 & 0.52 & $0.30-0.90$ & .02 \\
\hline Postoperative care & 1.14 & $0.73-1.77$ & .57 & 1.14 & $0.73-1.77$ & .57 \\
\hline Transfer from ward & 1.26 & $0.99-1.62$ & .06 & 1.26 & $0.99-1.62$ & 1.26 \\
\hline Vasoactives within $24 \mathrm{~h}$ & 1.41 & $1.02-1.95$ & .04 & 1.42 & $1.03-1.95$ & .03 \\
\hline Ventilation within $24 \mathrm{~h}$ & 1.19 & $0.82-1.74$ & .35 & 1.19 & $0.82-1.73$ & .36 \\
\hline
\end{tabular}

* Natural logarithmic transformation of PRISM III 24 risk of death.

† Natural logarithmic transformation of PRISM III 24 risk of death squared.

$\ddagger$ Previous ICU admission during current hospitalization. 
TABLE 4. Results of Multivariate Hierarchical, Logistic Regression Predicting Death Within 48 Hours After Admission for Diagnoses With $>10$ Deaths* $^{*}$

\begin{tabular}{|c|c|c|c|c|c|c|}
\hline & \multicolumn{3}{|c|}{ Weekend Admissions } & \multicolumn{3}{|c|}{ Evening Admissions } \\
\hline & OR & $95 \%$ CI & $P$ & OR & $95 \%$ CI & $P$ \\
\hline Cancer & 1.02 & $0.22-4.74$ & .98 & 0.76 & $0.21-2.66$ & .66 \\
\hline Cardiac arrest & 1.28 & $0.72-2.27$ & .84 & 1.80 & $1.04-3.13$ & .04 \\
\hline Congenital cardiovascular disease & 0.88 & $0.28-2.76$ & .83 & 3.90 & $1.37-11.07$ & .01 \\
\hline Acute HIE & 1.11 & $0.80-1.27$ & .96 & 1.43 & $0.24-8.60$ & .70 \\
\hline Meningitis & 0.97 & $0.29-3.29$ & .97 & 1.96 & $0.56-7.34$ & .28 \\
\hline Pneumonia/bronchiolitis & 1.47 & $0.57-3.81$ & .42 & 0.77 & $0.30-1.97$ & .59 \\
\hline Sepsis & 1.02 & $0.36-2.95$ & .96 & 1.47 & $0.53-4.10$ & .46 \\
\hline Shock & 0.48 & $0.21-1.11$ & .09 & 4.09 & $1.65-10.1$ & $<.01$ \\
\hline Trauma & 0.98 & $0.64-1.50$ & .93 & 1.03 & $0.66-1.63$ & .88 \\
\hline
\end{tabular}

* Diagnoses with $<10$ deaths included asthma, diabetic ketoacidosis, drug overdoses, acquired cardiovascular disease, medical device malfunctions, seizures, and suicide attempts. HIE, hypoxic-ischemic encephalopathy.

during the daytime hours (OR: 1.28; 95\% CI: $1.00-$ 1.62), after adjustment for the severity of illness and other covariates (Table 3). In the subgroup analyses (Table 4), we found higher odds of death among patients admitted with shock (OR: 4.09 ; $95 \%$ CI: $1.65-$ 10.1), with congenital cardiovascular disease (OR: 3.90; 95\% CI: 1.37-11.1), or after cardiac arrest (OR: 1.80; 95\% CI: 1.04-3.13). Other diagnosis-specific analyses did not demonstrate significantly different mortality rates when evening admissions were compared with daytime admissions.

\section{DISCUSSION}

In our investigation of 20547 patients admitted to the PICU on an emergency basis, there was no difference in the odds of death within 48 hours after admission between patients admitted on weekends and patients admitted on weekdays, after adjustment for the severity of illness. However, there was a $28 \%$ higher odds of death within 48 hours after admission when patients were admitted during evening hours, compared with daytime hours. The subgroup analyses revealed higher odds of death particularly among patients admitted with shock (OR: 4.09 ; 95\% CI: $1.65-$ 10.1), with congenital cardiovascular disease (OR: 3.90; 95\% CI: 1.37-11.1), or after cardiac arrest (OR: 1.80; $95 \%$ CI: $1.04-3.13)$.

Our finding of higher odds of death among nighttime admissions is consistent with similar studies in other patient populations. ${ }^{17-20}$ Heller et $\mathrm{al}^{21}$ performed a large (>350 000 births) observational study in Germany, in which "low-risk" infants born at night (9 PM to $7 \mathrm{AM}$ ) were twice as likely to die (OR: 1.86; 95\% CI: $1.10-3.13)$ than were those born during the daytime (7 AM to 9 PM). Similarly, higher odds of nighttime perinatal death (OR: 2.18; 95\% CI: 1.373.47) was found in a study of $>100000$ births in Wales. ${ }^{22}$ Stephansson et $\mathrm{al}^{18}$ also noted an increased risk of early neonatal death among nighttime births, after adjustment for the type of delivery and other confounders. Finally, in a recent, single-center, retrospective study of 6034 adult-patient admissions to an ICU, increased mortality rates were noted among those admitted at night. ${ }^{23}$

It is noteworthy that the increased risk of death among nighttime admissions was evident only after the analyses were adjusted for the severity of illness and that the increased risk of death was found pri- marily among 3 diagnosis subgroups. The former observation is an important methodologic consideration for the design and comparison of studies and depends on the quality of the tool used to assess the severity of illness. The PRISM III risk of death score is ideally suited for this purpose. For instance, in our database, patients with shock, congenital cardiovascular disease, or cardiac arrest represented 3 of the 5 diagnoses with the highest mean PRISM III scores and highest PRISM III predicted mortality rates. It remains important to prospectively test whether this observation results from differences between daytime and nighttime admission with respect to structure of care (staffing), process of care (behavior, fatigue), or both.

Our analysis did not identify an increased risk of death among pediatric patients admitted on weekends, compared with those admitted on weekdays, nor did the subgroup analysis reveal different outcomes for the 16 specific diagnoses evaluated. These results are also consistent with a recent study of neonatal mortality rates that used multivariate logistic regression to adjust for the severity of illness and the case mixture. ${ }^{24}$ Although earlier neonatal studies noted higher weekend mortality rates, those analyses likely did not adjust adequately for potential confounders. $4,6-8$

Interestingly, the lack of a weekend effect in the current study and the recent neonatal study ${ }^{24}$ differs from the results observed by Bell and Redelmeier, ${ }^{1}$ who evaluated mortality rates among adult emergency admissions between 1988 and 1997 in Ontario, Canada. The difference between these findings may represent differences between adult and pediatric patient populations or a distinction between the structure or processes of health care for adult versus pediatric patients. Alternatively, the disparity may result from differences in the ability of the PRISM III probability of death score and the Charlson comorbidity index used in the latter study to adequately adjust for the severity of illness. PRISM III mortality predictions are based on detailed, patient-level, demographic, physiologic, and laboratory data, whereas the Charlson comorbidity index relies on International Classification of Diseases categories. ${ }^{25,26}$ The use of administrative databases without physiologic or laboratory data has been shown to 
adjust inadequately for the severity of illness and the risk of death. ${ }^{14,15}$

There are limitations of the present study. First, deaths before admission to the PICU were not included in the analysis. Exclusion of deaths that occurred either "in the field" or in the emergency department could have underestimated the mortality rates for certain primary diagnoses such as trauma. ${ }^{27}$ Second, specific information on family preferences regarding therapy or refusal of certain treatment modalities, which could affect patient outcomes, was absent from the database. Third, PICU-specific information regarding differences in staff and physician coverage was not present in the database and might independently influence mortality rates. ${ }^{28,29}$ Last, the 15 PICUs in our analysis may not be nationally representative.

It remains important to determine the factors underlying the association between the time of admission and mortality rates as well as apparent differences between the results of recent reports. For instance, in the present study there were $28 \%$ more admissions per hour during evening hours, compared with daytime hours, and $6 \%$ fewer admissions per hour during weekend hours, compared with weekday hours. It is unclear whether the present findings are attributable to the observed admission densities or the increased human-error rates during evening hours, as reported in both medical and nonmedical fields. ${ }^{30-33}$ Nevertheless, it is possible to assess prospectively whether changes in the structure of care, process of care, or a combination thereof could negate the observed differences in mortality rates between daytime and nighttime admissions to the PICU.

\section{CONCLUSIONS}

Independent data for pediatric, neonatal, and adult patients now identify an association between the time of admission and mortality rates. Although the current study found no association between mortality rates and the day of admission, pediatric patients admitted to the PICU during evening hours had higher odds of death than did those admitted during daytime hours. It remains important to discern the basis of these observations (which will remain an important area of investigation) to maintain a consistent high quality of medical care.

\section{ACKNOWLEDGMENT}

This work was supported in part by the University of California, Davis, Children's Hospital Children's Miracle Network.

\section{REFERENCES}

1. Bell CM, Redelmeier DA. Mortality among patients admitted to hospitals on weekends as compared with weekdays. N Engl J Med. 2001;345: 663-668

2. Barnett MJ, Kaboli PJ, Sirio CA, Rosenthal GE. Day of the week of intensive care admission and patient outcomes: a multisite regional evaluation. Med Care. 2002;40:530-539

3. Stanley FJ, Alberman EV. Infants of very low birthweight: part I: perinatal factors affecting survival. Dev Med Child Neurol. 1978;20:300-312
4. Mangold WD. Neonatal mortality by the day of the week in the 1974-75 Arkansas live birth cohort. Am J Public Health. 1981;71:601-605

5. MacFarlane A. Variations in number of births and perinatal mortality by day of week in England and Wales. Br Med J. 1978;2:1670-1673

6. Mathers CD. Births and perinatal deaths in Australia: variations by day of week. J Epidemiol Community Health. 1983;37:57-62

7. Dowding VM, Duignan NM, Henry GR, MacDonald DW. Induction of labour, birthweight and perinatal mortality by day of the week. $\mathrm{Br} \mathrm{J}$ Obstet Gynaecol. 1987;94:413-419

8. Hendry RA. The weekend: a dangerous time to be born? Br J Obstet Gynaecol. 1981;88:1200-1203

9. Pollack MM, Patel KM, Ruttimann UE. PRISM III: an updated Pediatric Risk of Mortality score. Crit Care Med. 1996;24:743-752

10. Pollack MM, Ruttimann UE, Getson PR. Pediatric risk of mortality (PRISM) score. Crit Care Med. 1988;16:1110-1116

11. Donabedian A. Evaluating the quality of medical care. Milbank Mem Fund Q. 1966;44(suppl):166-206

12. Carcillo JA, Davis AL, Zaritsky A. Role of early fluid resuscitation in pediatric septic shock. JAMA. 1991;266:1242-1245

13. Marcin JP, Pollack MM. Review of the methodologies and applications of scoring systems in neonatal and pediatric intensive care. Pediatr Crit Care Med. 2000;1:20-27

14. Laupacis A, Sekar N, Stiell IG. Clinical prediction rules: a review and suggested modifications of methodological standards. JAMA. 1997;277: 488-494

15. Harrell FE Jr, Lee KL, Mark DB. Multivariable prognostic models: issues in developing models, evaluating assumptions and adequacy, and measuring and reducing errors. Stat Med. 1996;15:361-387

16. Leyland AH, Goldstein H. Multilevel Modelling of Health Statistics. New York, NY: Wiley; 2001

17. Domenighetti G, Paccaud F. The night: a dangerous time to be born? Br J Obstet Gynaecol. 1986;93:1262-1267

18. Stephansson O, Dickman PW, Johansson AL, Kieler H, Cnattingius S. Time of birth and risk of intrapartum and early neonatal death. Epidemiology. 2003;14:218-222

19. Paccaud F, Martin-Beran B, Gutzwiller F. Hour of birth as a prognostic factor for perinatal death. Lancet. 1988;1:340-343

20. Ruffieux C, Marazzi A, Paccaud F. The circadian rhythm of the perinatal mortality rate in Switzerland. Am J Epidemiol. 1992;135:936-952

21. Heller G, Misselwitz B, Schmidt S. Early neonatal mortality, asphyxia related deaths, and timing of low risk births in Hesse, Germany, 1990-8: observational study. BMJ. 2000;321:274-275

22. Stewart JH, Andrews J, Cartlidge PH. Numbers of deaths related to intrapartum asphyxia and timing of birth in all Wales perinatal survey, 1993-5. BMJ. 1998;316:657-660

23. Morales IJ, Peters SG, Afessa B. Hospital mortality rate and length of stay in patients admitted at night to the intensive care unit. Crit Care Med. 2003;31:858-863

24. Gould JB, Qin C, Marks AR, Chavez G. Neonatal mortality in weekend vs weekday births. JAMA. 2003;289:2958-2962

25. Deyo RA, Cherkin DC, Ciol MA. Adapting a clinical comorbidity index for use with ICD-9-CM administrative databases. J Clin Epidemiol. 1992; 45:613-619

26. Charlson ME, Pompei P, Ales KL, MacKenzie CR. A new method of classifying prognostic comorbidity in longitudinal studies: development and validation. J Chronic Dis. 1987;40:373-383

27. Carmody IC, Romero J, Velmahos GC. Day for night: should we staff a trauma center like a nightclub? Am Surg. 2002;68:1048-1051

28. Pollack MM, Patel KM, Ruttimann E. Pediatric critical care training programs have a positive effect on pediatric intensive care mortality. Crit Care Med. 1997;25:1637-1642

29. Pollack MM, Cuerdon TT, Patel KM, Ruttimann UE, Getson PR, Levetown M. Impact of quality-of-care factors on pediatric intensive care unit mortality. JAMA. 1994;272:941-946

30. Selbst SM, Fein JA, Osterhoudt K, Ho W. Medication errors in a pediatric emergency department. Pediatr Emerg Care. 1999;15:1-4

31. Borland RG, Rogers AS, Nicholson AN, Pascoe PA, Spencer MB. Performance overnight in shiftworkers operating a day-night schedule. Aviat Space Environ Med. 1986;57:241-249

32. Gillberg M, Kecklund G, Akerstedt T. Sleepiness and performance of professional drivers in a truck simulator: comparisons between day and night driving. J Sleep Res. 1996;5:12-15

33. Parks DK, Yetman RJ, McNeese MC, Burau K, Smolensky MH. Daynight pattern in accidental exposures to blood-borne pathogens among medical students and residents. Chronobiol Int. 2000;17:61-70 